\title{
ASO Visual Abstract: Pleural Invasion Depth of Disseminated Nodules in Patients with Stage IVa or Recurrent Thymoma: Assessment, Curative Impact, and Surgical Outcomes
}

\author{
Shota Nakamura, MD' ${ }^{1}$, Hisashi Tateyama, $\mathrm{MD}^{2}$, Keita Nakanishi, $\mathrm{MD}^{1}$, Tomoshi Sugiyama, $\mathrm{MD}^{\mathbf{1}}$, \\ Yuka Kadomatsu, MD ${ }^{1}$, Harushi Ueno, MD ${ }^{1}$, Masaki Goto, ${ }^{1}{ }^{1}$, Naoki Ozeki, MD $^{1}$, Takayuki Fukui, MD $^{1}$, \\ Kohei Yokoi, MD ${ }^{1}$, and Toyofumi F. Chen-Yoshikawa, MD ${ }^{1}$ \\ ${ }^{1}$ Department of Thoracic Surgery, Nagoya University Graduate School of Medicine, Nagoya, Japan; ${ }^{2}$ Department of \\ Pathology, Clinical Laboratory, Kasugai Municipal Hospital, Kasugai, Japan
}

Thymoma patients with pleural dissemination are difficult to manage and their treatment strategy remains undefined. The purpose of this study (https://doi.org/10. 1245/s10434-021-10888-0) was to investigate the clinicopathologic features of these patients, focusing on the association between the depth of pleural invasion and prognosis. Depth of pleural invasion may influence survival outcomes in thymoma patients with pleural dissemination. Patients with Da disseminated nodules had an increased probability of prolonged OS and PFS, tended to achieve negative PSM compared with patients with $\mathrm{Db}$.

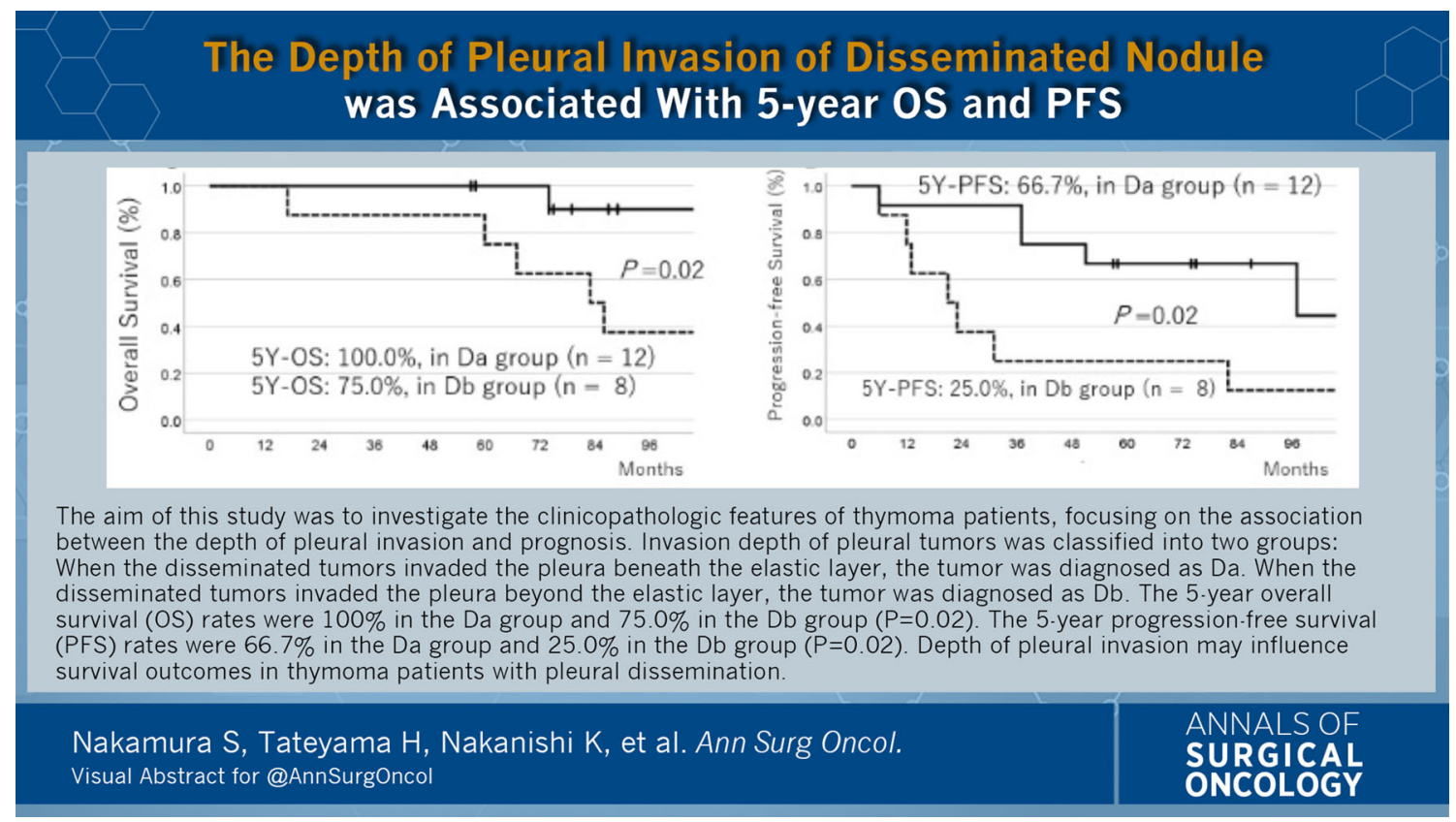

DISCLOSURES Part of this work was presented at the 18th World Conference of Lung Cancer, Yokohama, Japan, October, 17, 2017.

(C) Society of Surgical Oncology 2021

S. Nakamura, MD

e-mail: shota197065@med.nagoya-u.ac.jp
Publisher's Note Springer Nature remains neutral with regard to jurisdictional claims in published maps and institutional affiliations. 